\title{
Microsatellite loci development for three catfish species from northwestern South a America
}

Correspondence:

Edna J. Márquez ejmarque@unal.edu.co

Submitted July 24, 2019

Accepted December 19, 2019

by Guillermo Orti

Published April 20, 2020

\section{${ }^{\oplus}$ Natalia Restrepo-Escobar ${ }^{1}$ and ${ }^{\oplus}$ Edna J. Márquez ${ }^{1}$}

The Neotropical catfish species Ageneiosus pardalis, Pimelodus grosskopfii, and Sorubim cuspicaudus are important fishery resources in Colombia that show historical declines in their capture. This study used next-generation sequencing with 454 FLX technology (Roche Applied Science) and bioinformatics analysis to develop between 18 and 24 microsatellite loci for these species. The novel microsatellite loci showed high values of polymorphic information content -PIC (A. pardalis: $0.601-0.903$, P. grosskopfii: $0.748-0.946$ and S. cuspicaudus: $0.383-$ $0.876)$, and the average number of alleles/locus ranged from 7-15 for $A$. pardalis, 9-30 for P. grosskopfii and 5-14 for S. cuspicaudus. The average observed and expected heterozygosities were respectively, $0.757 \pm 0.035$ and $0.834 \pm 0.015$ for A. pardalis; $0.596 \pm 0.040$ and $0.881 \pm 0.009$ for P. grosskopfii; and $0.747 \pm 0.031$ and $0.757 \pm 0.025$ for $S$. cuspicaudus. For future studies, these loci can be useful to estimate the genetic diversity and population structure in these three Neotropical catfishes.

Keywords: Freshwater fish, Molecular markers, Next-generation sequencing, Siluriformes
Online version ISSN 1982-0224

Print version ISSN 1679-6225

Neotrop. Ichthyol.

vol. 18, no. 1, Maringá 2020

Epub, Apr 17, 2020

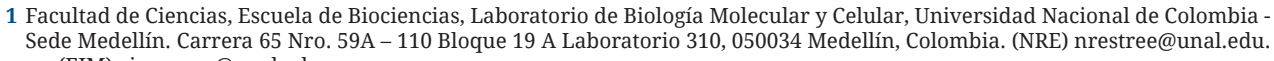
co, (EJM) ejmarque@unal.edu.co, 
Las especies de bagres neotropicales Ageneiosus pardalis, Pimelodus grosskopfii y Sorubim cuspicaudus, son importantes recursos pesqueros en Colombia y han mostrado disminuciones históricas en sus capturas. En este estudio se empleó la secuenciación genómica de próxima generación y análisis bioinformático para desarrollar entre 18 y 24 loci microsatélites para estas especies. Los loci microsatélites mostraron altos valores del contenido de información polimórfica CIP (A. pardalis: $0.601-0.903$, P. grosskopfii: $0.748-0.946$ and S. cuspicaudus: 0.383-0.876) y el número promedio de alelos/locus mostró un rango de 7-15 para $A$. pardalis, 9-30 para P. grosskopfii y 5-14 para S. cuspicaudus. Los valores promedio de heterocigosidad observada y esperada fueron respectivamente $0.757 \pm 0.035$ y $0.834 \pm 0.015$ para $A$. pardalis; $0.596 \pm 0.040$ y $0.881 \pm 0.009$ para P. grosskopfii; y $0.747 \pm 0.031$ y $0.757 \pm 0.025$ para $S$. cuspicaudus. Los loci microsatélites desarrollados en este trabajo pueden ser útiles para estimar la diversidad genética y la estructura poblacional de estos tres bagres neotropicales en estudios futuros.

Palabras clave: Peces de agua dulce, Marcadores Moleculares, Secuenciación de próxima generación, Siluriformes.

\section{INTRODUCTION}

Genetic population studies are crucial in the generation of valuable information for different programs of management, conservation, and the genetic-diversity monitoring of several species (Schwartz et al., 2007; Allendorf et al., 2010; Frankham, 2010); particularly, those affected by different anthropogenic activities (Frankham, 2010). Among the different molecular markers utilized in genetic population studies, microsatellite loci are one of the most informative and widely used (Hamilton et al., 1999; Guichoux et al., 2011). However, the first approaches for microsatellite loci development in non-model species were expensive, complex (Hamilton et al., 1999; Castoe et al., 2010; Fernandez-Silva et al., 2013), and produced a low number of useful markers obtained for population studies (Zalapa et al., 2012). Fortunately, nextgeneration sequencing technologies allowed the fast development of different useful molecular markers to generate population and evolutionary information of species at lower costs (Ekblom, Galindo, 2011; Guichoux et al., 2011; Fernandez-Silva et al., 2013; Miller et al., 2013), although for the vast majority of fish species these markers are still limited or absent (Kumar, Kocour, 2017).

In Siluriformes, one of the richest taxonomic order of freshwater fishes in the Neotropics (Pereira et al., 2013; Reis et al., 2016), microsatellite loci have been developed only for 19 of 2,315 Neotropical valid species. Pimelodidae, the most studied family, includes 12 species belonging to the genera Brachyplatystoma (Rodrigues et al., 2009; Batista et al., 2010); Conorhynchos (Carvalho, Beheregaray, 2011), Phractocephalus (Souza et al., 2012), Pimelodella (Moeser, Bermingham, 2005), Pimelodus (Paiva, Kalapothakis, 2008; see Agostini et al., 2011), Pseudoplatystoma (Revaldaves et al., 2005; Saulo- 
Machado et al., 2011; Prado et al., 2014), Steindachneridion (see Ojeda et al., 2016), and Zungaro (Carrillo-Ávila et al., 2009). Remaining studies includes three species of Loricariidae (Telles et al., 2010; Pereira et al., 2012; Galindo et al., 2015), two species of Trichomycteridae (Zamudio et al., 2009; Muñoz-Rojas et al., 2012), one species of Heptapteridae (Rodrigues et al., 2015) and one species of Pseudopimelodidae (SouzaShibatta et al., 2013).

Microsatellite loci are absent for catfishes from the west of the Eastern Cordillera of the Andes excepting Pimelodus grosskopfii (Hernandez-Escobar et al., 2011 in Agostini et al., 2011), limiting the population genetic studies for these species. Some authors have used microsatellite loci developed for close phylogenetically related species (heterologous loci); however, in some cases their use seems to be related to failures in the amplification, low levels of polymorphism, size homoplasy, null-alleles, and the amplification of non-orthologous loci (Primmer et al., 2005; Barbará et al., 2007; Castoe et al., 2010; Yue et al., 2010). This has stimulated the development of new molecular markers suitable for their population genetic studies.

Consequently, based on next-generation sequencing with the 454 GS-FLX technology (Roche Applied Science) and bioinformatics analysis, this study developed species-specific microsatellite loci for the non-model catfish species Ageneiosus pardalis (Lütken, 1874), Pimelodus grosskopfii (Steindachner, 1879), and Sorubim cuspicaudus (Littmann, Burr, Nass, 2000). These three carnivorous and migratory species are important for fisheries and many aspects of their basic biology and population genetics remain unknown, restraining the development of adequate management programs. This issue is important since population densities of these species have been decreased by anthropic activities in all Colombian watersheds (Galvis and Mojica, 2007; UsmaOviedo et al., 2009; Mojica et al., 2012), which led to their classification as vulnerable in the red list of freshwater fish of Colombia (Mojica et al., 2012). Moreover, P. grosskopfii was also included as a critically endangered species in the Red List of Threatened Species of the International Union for the Conservation of Nature (IUCN; Villa-Navarro et al., 2016). These tools will allow for future population genetic studies that support different proposals focused on the sustainable management and conservation of these species.

\section{MATERIAL AND METHODS}

Samples were collected from 2011 to 2014 in the lower section of the Cauca River and supplied to the Laboratorio de Biología Molecular y Celular (Universidad Nacional de Colombia), through the scientific cooperation agreement CT-2013-002443; framed in the environmental license \# 0155 of January 30, 2009 from Ministerio de Ambiente, Vivienda y Desarrollo Territorial. For each species, we took advantage of nuclear reads from pyrosequenced-genomic libraries of one individual collected in the lower section of the Cauca River (Restrepo-Escobar et al., 2016a,b). Identification of microsatellite loci, primer design, and electronic PCR to guarantee the correct alignment of primers were performed using the software and procedures used by Landínez-García, Márquez (2016). About 39 and 43 pairs from the list of primers validated by electronic PCR were selected to evaluate their consistent amplification and polymorphism under standard PCR conditions in 12 individuals from each species. Then, we selected pairs of primers 
that fulfilled the conditions proposed by Landínez-García, Márquez (2016): (1) specific amplification in all individuals within the sizes that were designed (100 to $350 \mathrm{bp}$ ), (2) band resolution, (3) specificity, and (4) ability to detect heterozygotes. The forward primers of these pairs were directly fluorescently labeled or universal markers were added to their $5^{\prime}$-tail to produce their fluorescent label through the three primer PCR method (Blacket et al., 2012) and were further evaluated in 50 individuals of each species.

The PCR amplification was carried out under the conditions proposed by LandínezGarcía, Márquez (2016) for the directly labeled primers, and by Landínez-García, Marquez (2018) for the primers marked using the three primer method. In both cases, the PCR products were separated in an ABI 3130 automatic sequencer (Applied Biosystems, USA) using GeneScan-500 LIZ (Applied Biosystems, USA) as the size marker; the electropherograms obtained were reviewed using GeneMapper 4.0 (Applied Biosystems, USA). Before the statistical analysis, Micro-Checker 2.2.1 (Van Oosterhout et al., 2004) was used to detect possible genotyping errors.

For each species, the genetic diversity, the allelic frequencies, the observed (Ho) and expected $(\mathrm{He})$ average heterozygosity and the average number of alleles per locus $(\mathrm{Na})$ were determined with the GenAlEx 6.503 (Peakall, Smouse, 2012). Additionally, Arlequin 3.5.2.2 (Excoffier, Lischer, 2010) was used to determine the statistical significance of the allelic frequencies in Hardy-Weinberg and Linkage equilibria. In the case of multiple comparisons, the statistical significance was adjusted by sequential Bonferroni correction (Rice, 1989). Furthermore, the polymorphic information content (PIC) was determined for each loci with CERVUS 3.0.7 (Marshall et al., 1998).

\section{RESULTS}

Genomic sequencing for $A$. pardalis generated 176,196 reads, 75,442 (43\%) contained microsatellite loci, 19,940 (11\%) potentially amplifiable loci (PAL) and 8,906 (5\%) were validated by electronic PCR. For P. grosskopfii, the genomic sequencing generated 138,830 reads, 47,438 (34\%) containing microsatellite loci, 23,937 (17\%) PAL and $12,542(9 \%)$ were validated by electronic PCR. Finally, the genomic sequencing for S. cuspicaudus generated a total of 123,158 reads, 43,302 (35\%) contained microsatellite loci; 16,428 (13\%) PAL and 8,840 (7\%) were validated by electronic PCR.

The three studied species showed higher diversity of microsatellite loci with 2-mer motifs $(57 \%-69 \%)$, followed by the 4-mer (15\%-23\%), 3-mer $(10 \%-12 \%)$, 5-mer $(4 \%-5 \%)$ and a small portion of 6 -mer motifs ( $2 \%-3 \%$; Fig. 1$)$. Additionally, the three species showed similar patterns in the abundance of specific repeat motifs. For 2-mer motifs, the most common recurrence pattern was AC, and the less common was CG. Among the 3-mer motifs, the most common was ATT, and the least common was TCG for the three species. Lastly, among the 4-mer, the five most common motifs were AAAT, ATCT, TCTG, AGTG, and AATG.

More than $68 \%$ of the evaluated loci generated fragments in the expected size range (100-350 bp) and were polymorphic in A. pardalis (27 loci), P. grosskopfii (27 loci), and S. cuspicaudus (37 loci). The PIC values for the microsatellite loci developed were 0.6010.903 for A. pardalis, 0.748-0.946 for P. grosskopfii, and 0.383-0.876 for S. cuspicaudus (Tabs. 1-3). The average number of alleles per locus ranged from 7-15 alleles/locus 


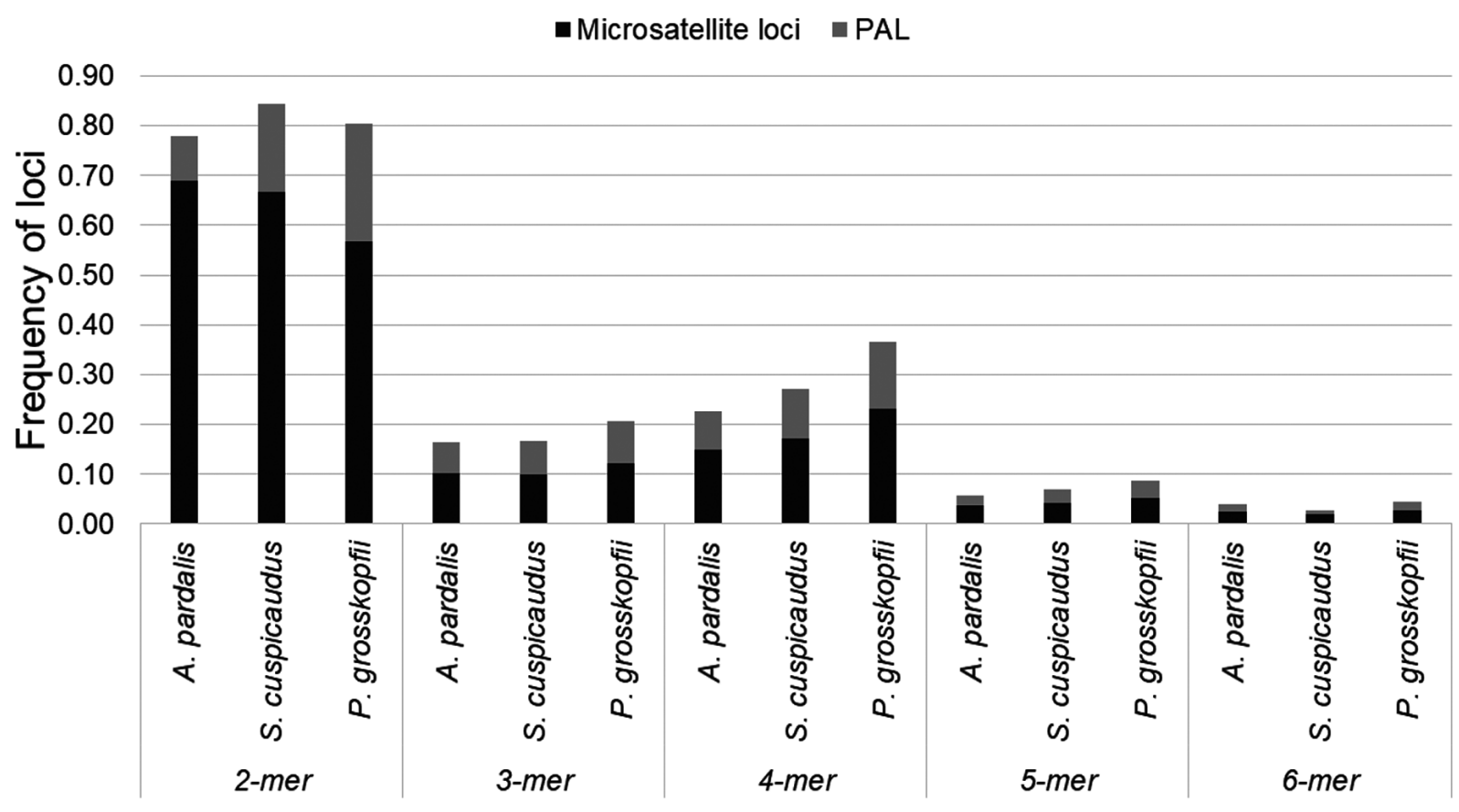

FIGURE 1 I Frequency of identified microsatellite loci and potentially amplifiable loci (PAL) obtained from 454 FLX sequencing for Ageneiosus pardalis, Pimelodus grosskopfii, and Sorubim cuspicaudus.

(average: $11.286 \pm 0.574$ ) in A. pardalis; 9-30 alleles/locus (average: $14.944 \pm 1.136$ ) in $P$. grosskopfii; and 5-14 alleles/locus (average: $9.500 \pm 0.552$ alleles/locus) in S. cuspicaudus. In addition, the expected average heterozygosity ranged from 0.622 to 0.910 in $A$. pardalis (He: $0.834 \pm 0.015$ ), 0.771-0.948 in P. grosskopfii (He: $0.878 \pm 0.010$ ), and 0.4190.887 in S. cuspicaudus (He: $0.757 \pm 0.025$ ). The observed average heterozygosity ranged from $0.320-0.920$ in A. pardalis (Ho: $0.757 \pm 0.035$ ), 0.340-0.920 in P. grosskopfii (Ho: $0.612 \pm 0.042$ ), and $0.300-0.940$ in S. cuspicaudus (Ho: $0.747 \pm 0.031$ ).

For A. pardalis (17 of 21, Tab. 1) and S. cuspicaudus (17 of 24, Tab. 3), all or most of the evaluated loci were shown to be in linkage and Hardy-Weinberg equilibria after the Bonferroni correction. In contrast, P. grosskopfii showed 13 loci with heterozygote deficit, allelic frequencies departures from Hardy-Weinberg equilibrium (Tab. 2), and significant linkage disequilibrium between the pairs of loci Pgrk01-Pgrk02 and Pgrk08-Pgrk20.

\section{DISCUSSION}

In this work, 63 microsatellite loci were designed for future studies of the genetic diversity of $A$. pardalis (21), S. cuspicaudus (24), and P. grosskopfii (18). The microsatellite loci of $A$. pardalis and $S$. cuspicaudus represent the first species-specific codominant markers for both Neotropical genera. Along with the above, the new 18 microsatellite loci for P. grosskopfii complement the currently available markers (Agostini et al., 2011). Pyrosequencing also 
TABLE 1 I Primer sequences and characteristics of 21 polymorphic microsatellite loci identified in Ageneiosus pardalis. Ra: Allelic size range; Na: Average number of alleles per locus; Ho and He: observed and expected heterozygosity, respectively; P: Statistical significance for tests of departure of Hardy-Weinberg equilibrium; PIC: Polymorphic information content. Annealing temperature: $56.5^{\circ} \mathrm{C}$, for all primers.

\begin{tabular}{|c|c|c|c|c|c|c|c|c|}
\hline Locus & Repeat motif & $\begin{array}{l}\text { Primer sequence for } \\
\text { forward }(F) \text { and reverse }(R)\left(5^{\prime} \rightarrow 3^{\prime}\right)\end{array}$ & $\mathbf{R a}$ & $\mathrm{Na}$ & Ho & $\mathrm{He}$ & $\mathbf{P}$ & PIC \\
\hline Apar03 & $(\mathrm{ATT})_{\mathrm{n}}$ & $\begin{array}{l}\text { F: TTTAGAAGCAGCCTGGATGG } \\
\text { R: CTTGACTTTGGGAAATGGGC }\end{array}$ & $186-219$ & 11 & 0.920 & 0.882 & 0.965 & 0.870 \\
\hline Apar25 & $(\mathrm{TCTG})_{\mathrm{n}}$ & $\begin{array}{l}\text { F: CCGGCTGTTCAATTTGTGG } \\
\text { R: CACAGAGTAGAAGAGCTCACTTTGG }\end{array}$ & 256-316 & 9 & 0.700 & 0.622 & 0.909 & 0.601 \\
\hline Apar11 & $(\mathrm{ATGG})_{\mathrm{n}}$ & $\begin{array}{l}\text { F: CACCAATGTATCCCCATCCC } \\
\text { R: TTCAAGTACTGGCTACAAGCTGC }\end{array}$ & 208-268 & 15 & 0.920 & 0.895 & 0.904 & 0.886 \\
\hline Apar36 & $(\text { AATAG })_{n}$ & $\begin{array}{l}\text { F: GAAGGAAATCACAGCCCTCG } \\
\text { R: TCTGCTGGAAGGTGGAGAGG }\end{array}$ & $230-280$ & 8 & 0.800 & 0.825 & 0.832 & 0.801 \\
\hline Apar18 & $(\mathrm{ATCT})_{\mathrm{n}}$ & $\begin{array}{l}\text { F: GCATCTGCACCTCATATTTGC } \\
\text { R: TGAGCTATTTCTGATTGGCTGG }\end{array}$ & 206-266 & 13 & 0.860 & 0.871 & 0.816 & 0.858 \\
\hline Apar05 & $(\mathrm{ATT})_{\mathrm{n}}$ & $\begin{array}{l}\text { F: GGGAGGGAGAGGAGGATAGC } \\
\text { R: GAAGGTAGGTGTTGGCAATGG }\end{array}$ & $192-234$ & 15 & 0.820 & 0.798 & 0.799 & 0.783 \\
\hline Apar35 & $(\text { AATAG })_{n}$ & $\begin{array}{l}\text { F: CCCTTTAAGCAAATTGTCTTCAGC } \\
\text { R: CACCATCTGTCCTGTCCTGC }\end{array}$ & 179-209 & 7 & 0.780 & 0.752 & 0.797 & 0.715 \\
\hline Apar22 & $(\mathrm{ATCT})_{\mathrm{n}}$ & $\begin{array}{l}\text { F: CAGAGGCTATTGTTCAGGCG } \\
\text { R: TCATTGGGGTGGTGATATGC }\end{array}$ & 235-271 & 10 & 0.800 & 0.850 & 0.612 & 0.833 \\
\hline Apar20 & $(\mathrm{ATCT})_{\mathrm{n}}$ & $\begin{array}{l}\text { F: TCCAACCATTACTGCATCCC } \\
\text { R: GGTGATCGTTGGATGATTTGC }\end{array}$ & 179-226 & 11 & 0.820 & 0.871 & 0.591 & 0.857 \\
\hline Apar21 & $(\mathrm{ATCT})_{\mathrm{n}}$ & $\begin{array}{l}\text { F: CACTGTGTGATCTCTCTGTGTTGG } \\
\text { R: GAGATATTTCTTGAATTTCCTTCGG }\end{array}$ & $173-225$ & 13 & 0.920 & 0.891 & 0.494 & 0.881 \\
\hline Apar27 & $(\mathrm{AATG})_{\mathrm{n}}$ & $\begin{array}{l}\text { F: TCACCAAAGTGAAGCTGTTGC } \\
\text { R: CAAACCAAGGACCTTCTCGC }\end{array}$ & $146-178$ & 9 & 0.900 & 0.827 & 0.416 & 0.806 \\
\hline Apar30 & $(\text { ATATC })_{n}$ & $\begin{array}{l}\text { F: GAACCTTGATTTTGCCCACG } \\
\text { R: GCCAACATCAGGAAAGGGG }\end{array}$ & $172-222$ & 11 & 0.820 & 0.844 & 0.414 & 0.827 \\
\hline Apar04 & $(\mathrm{ATC})_{\mathrm{n}}$ & $\begin{array}{l}\text { F: CCTGCACTTCAACACTTCACC } \\
\text { R: GAGAGAGTAAATGAGGGAAAGCG }\end{array}$ & $256-298$ & 10 & 0.880 & 0.844 & 0.401 & 0.826 \\
\hline Apar34 & $(\operatorname{ATCAC})_{n}$ & $\begin{array}{l}\text { F: ACACTGCATCCCATCACACC } \\
\text { R: TCCTCCTGGTCTTCTCACAGG }\end{array}$ & $191-251$ & 12 & 0.800 & 0.809 & 0.386 & 0.786 \\
\hline Apar23 & $(\mathrm{ATCT})_{\mathrm{n}}$ & $\begin{array}{l}\text { F: TCTTTGGTAACCCACCACCC } \\
\text { R: ATGTGCAATGGGGATCTGC }\end{array}$ & 241-269 & 8 & 0.860 & 0.814 & 0.295 & 0.791 \\
\hline Apar28 & $(\mathrm{ATGG})_{\mathrm{n}}$ & $\begin{array}{l}\text { F: AACTCCATGTCTGCTTCGGC } \\
\text { R: CTGCTATTGCAGCCCATCC }\end{array}$ & 273-301 & 8 & 0.600 & 0.698 & 0.179 & 0.666 \\
\hline Apar12 & $(\mathrm{ATCT})_{\mathrm{n}}$ & $\begin{array}{l}\text { F: GATTCCAGGATGCAGTTGAGG } \\
\text { R: AGCGATTGCACACCATAACC }\end{array}$ & $247-299$ & 14 & 0.780 & 0.886 & 0.129 & 0.876 \\
\hline Apar31 & $(\text { AATAG })_{n}$ & $\begin{array}{l}\text { F: CTGGAGGGATGCAAACTGC } \\
\text { R: GGGAATTCCGTTATTTTCAAGC }\end{array}$ & $151-236$ & 10 & 0.560 & 0.843 & 0.000 & 0.825 \\
\hline Apar14 & $(\mathrm{ATCT})_{\mathrm{n}}$ & $\begin{array}{l}\text { F: TTCCGGTATCGTGCATTTCC } \\
\text { R: AACAATTGGGTGCCTAAGACG }\end{array}$ & $165-221$ & 15 & 0.500 & 0.910 & 0.000 & 0.903 \\
\hline Apar32 & $(\text { ATTAG })_{n}$ & $\begin{array}{l}\text { F: TTGCACTTCTGGTTGGATGC } \\
\text { R: TCCAAAGCAAATGGTCATGG }\end{array}$ & 151-216 & 13 & 0.540 & 0.900 & 0.000 & 0.892 \\
\hline Apar19 & $(\mathrm{ATCT})_{\mathrm{n}}$ & $\begin{array}{l}\text { F: TCAGCTAAGGCAAGTTGTTTGC } \\
\text { R: GGGATTTCCTATATCGGCAGC }\end{array}$ & $190-250$ & 15 & 0.320 & 0.885 & 0.000 & 0.874 \\
\hline $\begin{array}{l}\text { Across } \\
\text { loci }\end{array}$ & & & & $11.286 \pm 0.574$ & $\begin{array}{l}0.757 \pm \\
0.035\end{array}$ & $\begin{array}{l}0.834 \pm \\
0.015\end{array}$ & 0.000 & 0.817 \\
\hline
\end{tabular}


TABLE 2 I Primer sequences and characteristics of 18 polymorphic microsatellite loci identified in Pimelodus grosskopfii. Ra: Allelic size range; Na: Average number of alleles per locus; Ho and He: observed and expected heterozygosity, respectively; P: Statistical significance for tests of departure of Hardy-Weinberg equilibrium; PIC: Polymorphic information content. ${ }^{1,2,3}$ Annealing temperatures: $56.5^{\circ} \mathrm{C}, 59^{\circ} \mathrm{C}, 60{ }^{\circ} \mathrm{C}$, respectively.

\begin{tabular}{|c|c|c|c|c|c|c|c|c|}
\hline Locus & Repeat motif & $\begin{array}{l}\text { Primer sequence for } \\
\text { forward }(F) \text { and reverse }(R)\left(5^{\prime} \rightarrow 3^{\prime}\right)\end{array}$ & $\mathbf{R a}$ & $\mathbf{N a}$ & Ho & $\mathrm{He}$ & $\mathbf{P}$ & PIC \\
\hline${ }^{1}$ Pgrk40 & $(\mathrm{ATCT})_{\mathrm{n}}$ & $\begin{array}{l}\text { F: TTTGGTAACATTCAAGGTTTACTTGC } \\
\text { R: AAAATAGCAACGTTCTAACTAGGGG }\end{array}$ & $159-219$ & 15 & 0.920 & 0.916 & 0.743 & 0.910 \\
\hline${ }^{1}$ Pgrk27 & $(\operatorname{ATATT})_{n}$ & $\begin{array}{l}\text { F: CTTCCAAGCATGACAAGCCC } \\
\text { R: CTCTCCACCACATCTTCCCC }\end{array}$ & 264-359 & 16 & 0.920 & 0.866 & 0.517 & 0.852 \\
\hline${ }^{1}$ Pgrk15 & $(\mathrm{ATCT})_{\mathrm{n}}$ & $\begin{array}{l}\text { F: TGCCATCAGTGGTCTTCACC } \\
\text { R: CCCCATCACCCTGTTCACC }\end{array}$ & $268-328$ & 14 & 0.760 & 0.852 & 0.225 & 0.840 \\
\hline${ }^{2}$ Pgrk14 & $(\mathrm{ATCT})_{\mathrm{n}}$ & $\begin{array}{l}\text { F: TCATGGCCTTGTACTTGTACCG } \\
\text { R: CACCAGTTCATGCTTCTGCC }\end{array}$ & $169-261$ & 15 & 0.800 & 0.903 & 0.170 & 0.895 \\
\hline${ }^{2}$ Pgrk03 & $(\mathrm{ATCT})_{\mathrm{n}}$ & $\begin{array}{l}\text { F: GGATGAAAGAAAAGGATTTGGC } \\
\text { R: GATAAGACGCGCTTACACTTGC }\end{array}$ & $174-246$ & 16 & 0.740 & 0.909 & 0.083 & 0.902 \\
\hline${ }^{2}$ Pgrk24 & $(\mathrm{AAAT})_{\mathrm{n}}$ & $\begin{array}{l}\text { F: ACACGCATGTCTCTTGCCC } \\
\text { R: ACTTGTACTGCGGATGCGG }\end{array}$ & $188-232$ & 11 & 0.660 & 0.866 & 0.016 & 0.852 \\
\hline${ }^{2}$ Pgrk01 & $(\mathrm{ATCT})_{\mathrm{n}}$ & $\begin{array}{l}\text { F: GTCCCAGTCCTGCTTCACG } \\
\text { R: GCTAATGGTACAACATCGCCC }\end{array}$ & $156-292$ & 30 & 0.760 & 0.948 & 0.002 & 0.946 \\
\hline${ }^{2}$ Pgrk19 & $(\mathrm{AAAT})_{\mathrm{n}}$ & $\begin{array}{l}\text { F: TAGTCGGTGCTAATTCGCCG } \\
\text { R: ACTGACTGGAGACCACAGCG }\end{array}$ & 201-237 & 10 & 0.520 & 0.810 & 0.000 & 0.787 \\
\hline${ }^{2}$ Pgrk12 & $(\mathrm{ATCT})_{\mathrm{n}}$ & $\begin{array}{l}\text { F: TGAGATTGGTTTATAACACAGACCG } \\
\text { R: TGCAGCTGAAGAACTCAGGG }\end{array}$ & $192-292$ & 22 & 0.380 & 0.913 & 0.000 & 0.908 \\
\hline${ }^{1}$ Pgrk18 & $(\mathrm{ATCT})_{\mathrm{n}}$ & $\begin{array}{l}\text { F: GGATAAATATGGGTGGGTGGC } \\
\text { R: GGAGCTGTGGAAGAGACATCG }\end{array}$ & $210-282$ & 15 & 0.580 & 0.907 & 0.000 & 0.900 \\
\hline${ }^{2}$ Pgrk07 & $(\mathrm{ATCT})_{\mathrm{n}}$ & $\begin{array}{l}\text { F: TGGCACATGAGTTGAAGACG } \\
\text { R: AAACACATTGACTGATAGCCTTCC }\end{array}$ & $197-265$ & 17 & 0.400 & 0.907 & 0.000 & 0.899 \\
\hline${ }^{3}$ Pgrk06 & $(\mathrm{ATCT})_{\mathrm{n}}$ & $\begin{array}{l}\text { F: GCGGGAAAAGTACAGGAAGG } \\
\text { R: CGACGCAGCTCAGAATAAAGC }\end{array}$ & $180-264$ & 13 & 0.520 & 0.899 & 0.000 & 0.890 \\
\hline${ }^{1}$ Pgrk31 & $(\mathrm{ATT})_{\mathrm{n}}$ & $\begin{array}{l}\text { F: TGGAATTGTGCATTTCTTTGC } \\
\text { R: GGAGGTTGAATTCCTCTGTGG }\end{array}$ & $154-208$ & 15 & 0.580 & 0.888 & 0.000 & 0.878 \\
\hline${ }^{2}$ Pgrk02 & $(\mathrm{ATCT})_{\mathrm{n}}$ & $\begin{array}{l}\text { F: ATCTGTCTGGCCATCCACC } \\
\text { R: CAGATAGACGGACGCACG }\end{array}$ & 77-157 & 15 & 0.400 & 0.879 & 0.000 & 0.867 \\
\hline${ }^{1}$ Pgrk20 & $(\mathrm{ATCT})_{\mathrm{n}}$ & $\begin{array}{l}\text { F: CAAGCTGCCTCCTGAAAACC } \\
\text { R: GCTCATCTGTGTGAGTGGTGC }\end{array}$ & $124-216$ & 14 & 0.600 & 0.868 & 0.000 & 0.855 \\
\hline${ }^{1}$ Pgrk10 & $(\mathrm{ATCT})_{\mathrm{n}}$ & $\begin{array}{l}\text { F: TGTTGCGTAATATTCCCAGGC } \\
\text { R: GACACAAACTGGTGAAAATCTGC }\end{array}$ & $230-282$ & 11 & 0.480 & 0.864 & 0.000 & 0.849 \\
\hline${ }^{2}$ Pgrk28 & $(\mathrm{AATAG})_{\mathrm{n}}$ & $\begin{array}{l}\text { F: ACTGTCTTGTGGCTTTGTGC } \\
\text { R: TACGGGAAAATAATCCTGGG }\end{array}$ & $116-226$ & 11 & 0.660 & 0.843 & 0.000 & 0.825 \\
\hline${ }^{2}$ Pgrk08 & $(\mathrm{AAAT})_{\mathrm{n}}$ & $\begin{array}{l}\text { F: TTGGCGTGCAAATGAATCC } \\
\text { R: ATGGGACCACAATCAGAGGC }\end{array}$ & 261-301 & 9 & 0.340 & 0.771 & 0.000 & 0.748 \\
\hline Across loci & & & & $14.944 \pm 1.136$ & $0.612 \pm 0.042$ & $0.878 \pm 0.010$ & 0.000 & 0.867 \\
\hline
\end{tabular}


TABLE 3 I Primer sequences and characteristics of 24 polymorphic microsatellite loci identified in Sorubim cuspicaudus. Ra: Allelic size range; Na: Average number of alleles per locus; Ho and He: observed and expected heterozygosity, respectively; P: Statistical significance for tests of departure of Hardy-Weinberg equilibrium; PIC: Polymorphic information content. Annealing temperature: $56.5^{\circ} \mathrm{C}$, for all primers.

\begin{tabular}{|c|c|c|c|c|c|c|c|c|}
\hline Locus & Repeat motif & $\begin{array}{l}\text { Primer sequence for } \\
\text { forward }(F) \text { and reverse }(R)\left(5^{\prime} \rightarrow 3^{\prime}\right)\end{array}$ & $\mathbf{R a}$ & $\mathbf{N a}$ & Ho & He & $\mathbf{P}$ & PIC \\
\hline Scus22 & $(\mathrm{ATCT})_{\mathrm{n}}$ & $\begin{array}{l}\text { F: CGCCACCTTAGGAACCTACC } \\
\text { R: GTGGTATGGTGGTGTCGAGG }\end{array}$ & $180-208$ & 8 & 0.800 & 0.808 & 0.932 & 0.784 \\
\hline Scus15 & $(\mathrm{TTC})_{\mathrm{n}}$ & $\begin{array}{l}\text { F: GCCTTCATATGCTTGGACCC } \\
\text { R: TGAAGGTCCCTTTCAATGGC }\end{array}$ & $201-216$ & 6 & 0.620 & 0.637 & 0.913 & 0.599 \\
\hline Scus41 & $(\text { ATTAC })_{n}$ & $\begin{array}{l}\text { F: CAGATAAAGGACCATGCTGAGG } \\
\text { R: CTGGATGCTCTGTGTGAGGC }\end{array}$ & $161-186$ & 6 & 0.780 & 0.689 & 0.895 & 0.636 \\
\hline Scus24 & $(\mathrm{ATCT})_{\mathrm{n}}$ & $\begin{array}{l}\text { F: CCTTGAGATACCTGCAGCCC } \\
\text { R: GTTCCCTCCCTTCGTCTTCC }\end{array}$ & $257-289$ & 9 & 0.820 & 0.831 & 0.847 & 0.809 \\
\hline Scus17 & $(\mathrm{ATCT})_{\mathrm{n}}$ & $\begin{array}{l}\text { F: TGATAGAGGCACAGAGTGAACG } \\
\text { R: CCTGTGTGCCTTGATGTCG }\end{array}$ & $181-229$ & 12 & 0.900 & 0.862 & 0.809 & 0.847 \\
\hline Scus43 & $(\text { ATTAG })_{n}$ & $\begin{array}{l}\text { F: TGACTCTGTGACTCTAATTCCTGC } \\
\text { R: TCAGTTTCCCTGAGGAACCC }\end{array}$ & $141-166$ & 6 & 0.720 & 0.629 & 0.809 & 0.568 \\
\hline Scus28 & $(\mathrm{ATCT})_{\mathrm{n}}$ & $\begin{array}{l}\text { F: TCGGATGCAAAGTTAGACGG } \\
\text { R: AGGATTTGTTGCCTCATGCC }\end{array}$ & $252-280$ & 8 & 0.820 & 0.803 & 0.794 & 0.774 \\
\hline Scus25 & $(\mathrm{ATCT})_{\mathrm{n}}$ & $\begin{array}{l}\text { F: TAAAACTGCAAGCCCСTTCG } \\
\text { R: CAGAGTCCAAACCCTGTGGG }\end{array}$ & $173-237$ & 14 & 0.920 & 0.867 & 0.746 & 0.853 \\
\hline Scus23 & $(\mathrm{ATCT})_{\mathrm{n}}$ & $\begin{array}{l}\text { F: GATCTTTGTTCAGTCCACGGC } \\
\text { R: GACGTTGTTAGCCCAGATGC }\end{array}$ & $275-309$ & 11 & 0.780 & 0.838 & 0.523 & 0.818 \\
\hline Scus12 & $(\mathrm{ATT})_{\mathrm{n}}$ & $\begin{array}{l}\text { F: TCTACACAAGCAGAAATGCAGC } \\
\text { R: TCACATTGCTTGACAGATGCC }\end{array}$ & $204-258$ & 11 & 0.680 & 0.695 & 0.514 & 0.670 \\
\hline Scus35 & $(\text { ATATT })_{n}$ & $\begin{array}{l}\text { F: GTCAAAACGCATTCTGTAAATGG } \\
\text { R: GTAGTGCTCAGCTCCAGGGC }\end{array}$ & $209-249$ & 9 & 0.880 & 0.832 & 0.479 & 0.812 \\
\hline Scus40 & $(\mathrm{ATCTT})_{\mathrm{n}}$ & $\begin{array}{l}\text { F: TGGAAGTAGTTGCTGCTGTTGC } \\
\text { R: CAGAAGGCATTTACTGTCTGGC }\end{array}$ & $241-276$ & 7 & 0.660 & 0.641 & 0.438 & 0.594 \\
\hline Scus20 & $(\mathrm{ATCT})_{\mathrm{n}}$ & $\begin{array}{l}\text { F: GTCACATCTGGGTGTGCTGG } \\
\text { R: GCCTGAGCTTTGGATTGAGC }\end{array}$ & $249-317$ & 14 & 0.940 & 0.887 & 0.321 & 0.876 \\
\hline Scus39 & $(\mathrm{ATCTT})_{\mathrm{n}}$ & $\begin{array}{l}\text { F: GGGACACTATGATTTTCCTTCAGC } \\
\text { R: CTGTCATCCCCAGCAGTAGC }\end{array}$ & $128-168$ & 9 & 0.820 & 0.812 & 0.199 & 0.785 \\
\hline Scus32 & $(A G G G C)_{n}$ & $\begin{array}{l}\text { F: AACGAGCCATGCAAGAACG } \\
\text { R: CCCAGAAGCAGTAAGGTGTGC }\end{array}$ & $222-287$ & 14 & 0.820 & 0.861 & 0.122 & 0.848 \\
\hline Scus21 & $(\mathrm{ATCT})_{\mathrm{n}}$ & $\begin{array}{l}\text { F: CTACCCAATCTAGCTGCCCC } \\
\text { R: CGACCTGGTGGGTACGTAGG }\end{array}$ & $117-221$ & 11 & 0.900 & 0.820 & 0.080 & 0.798 \\
\hline Scus03 & $(\mathrm{ATC})_{\mathrm{n}}$ & $\begin{array}{l}\text { F: GGATCGTTGGTGTAAATCTCTGC } \\
\text { R: TGACTCATGGTGTGTGGTGC }\end{array}$ & $114-135$ & 7 & 0.520 & 0.575 & 0.052 & 0.536 \\
\hline Scus11 & $(\mathrm{ATT})_{\mathrm{n}}$ & $\begin{array}{l}\text { F: CACTTCCACCTGAACCATGC } \\
\text { R: GCATCAGGGTTAGCACAGGC }\end{array}$ & $276-288$ & 5 & 0.300 & 0.419 & 0.037 & 0.383 \\
\hline Scus16 & $(\mathrm{AATG})_{\mathrm{n}}$ & $\begin{array}{l}\text { F: ACGTCATCTGGTGTCGTTGG } \\
\text { R: CCAGAAGATCTGTGTGAACCG }\end{array}$ & $104-136$ & 9 & 0.620 & 0.780 & 0.026 & 0.748 \\
\hline Scus13 & $(\mathrm{ATT})_{\mathrm{n}}$ & $\begin{array}{l}\text { F: AGACGAGAGGGGTGACATGC } \\
\text { R: AAGGTGTTGGCAGGATGAGG }\end{array}$ & $272-314$ & 9 & 0.740 & 0.809 & 0.024 & 0.783 \\
\hline Scus10 & $(\mathrm{ATT})_{\mathrm{n}}$ & $\begin{array}{l}\text { F: CCTGAAGAAATCCAGCCACC } \\
\text { R: ACCACAACTCCGACTCCAGG }\end{array}$ & $168-213$ & 13 & 0.820 & 0.865 & 0.020 & 0.851 \\
\hline Scus18 & $(\mathrm{ATCT})_{\mathrm{n}}$ & $\begin{array}{l}\text { F: CAAGCATCAAAGCAGAAAGCC } \\
\text { R: ATCACCATCTTGCGTTCTATCC }\end{array}$ & $257-305$ & 12 & 0.640 & 0.867 & 0.005 & 0.853 \\
\hline Scus37 & $(\text { ATCAG })_{n}$ & $\begin{array}{l}\text { F: GATCTGGGCTCTGCTTTTGG } \\
\text { R: GCAGCACAATGAGTACTGCG }\end{array}$ & $265-330$ & 9 & 0.540 & 0.597 & 0.002 & 0.564 \\
\hline Scus07 & $(\mathrm{ATT})_{\mathrm{n}}$ & $\begin{array}{l}\text { F: CAACATCTGCAACCAAATGC } \\
\text { R: GATTTTGCACCAGGACAACG }\end{array}$ & $146-176$ & 9 & 0.880 & 0.753 & 0.000 & 0.713 \\
\hline $\begin{array}{c}\text { Across } \\
\text { loci }\end{array}$ & & & & $9.500 \pm 0.552$ & $0.747 \pm 0.031$ & $0.757 \pm 0.025$ & 0.000 & 0.729 \\
\hline
\end{tabular}


allowed us to examine the richness of microsatellite motifs of the species and compare them with the information available for eukaryotic organisms (Meglécz et al., 2012), particularly bony fishes (Osteichthyes) of the order Siluriformes (Somridhivej et al., 2008; Mohindra et al., 2012; Zhang et al., 2014), Characiformes (Villanova et al., 2015; Yazbeck et al., 2018), Cypriniformes (Luo et al., 2012; Jorge et al., 2018), Perciformes (Saarinen, Austin, 2010), and Tetraodontiformes (Edwards et al., 1998).

Superficial sequencing on A. pardalis, P. grosskopfii, and S.cuspicaudus, showed a higher frequency of 2-mer motifs; a characteristic previously described for several eukaryotic organisms (Meglécz et al., 2012). Additionally, in this study, 4-mer are the second most frequent motif for the three species studied, a similar outcome to others fishes such as Megaleporinus obtusidens (=Leporinus obtusidens in Villanova et al., 2015); Craterocephalus fluviatilis, Galaxias fuscus, Henicorhynchus lobatus, Henicorhynchus siamensis, Alticus arnoldorum, Amphiprion sandaracinos and Amphiprion mccullochi (Meglécz et al., 2012). This result is in contrast however, to others species of fishes such as Ictalurus punctatus (Somridhivej et al., 2008), Clarias batrachus (Mohindra et al., 2012), Tachysurus fulvidraco (=Pelteobagrus fulvidraco in Zhang et al., 2014), Brycon orbignyanus (Yazbeck et al., 2018) and Schizothorax biddulphi (Luo et al., 2012), which exhibit 3-mer as the second most frequent motif.

The high frequency of the AC repeat motif is concordant with that described for all the Chordata phylum species, especially for the species of the Actinopterygii class (Meglécz et al., 2012). Similarly, the low frequency of the CG repeat motif found in this work is consistent with that described for most eukaryotic species (Meglécz et al., 2012). The most common repeat motifs found in this work, AC and ATT, have been described in bony fishes such as Rhamdia sp. (Rodrigues et al., 2015), I. punctatus (Somridhivej et al., 2008), C. batrachus (Mohindra et al., 2012), T. fulvidraco (Zhang et al., 2014), M. obtusidens (Villanova et al., 2015), S. biddulphi (Luo et al., 2012), and Etheostoma okaloosae (Saarinen, Austin, 2010). However, the frequency of the ATT motif differs from that found in Piaractus brachypomus (AGC, Jorge et al., 2018) and Takifugu rubripes (AGG; Edwards et al., 1998). The most frequent 4-mer motifs repeat found for the three studied catfish species (AAAT, ATCT, TCTG, AGTG, and AATG) are also among the most frequent for other catfishes, such as I. punctatus (Somridhivej et al., 2008), C. batrachus (Mohindra et al., 2012) and T. fulvidraco (Zhang et al., 2014), as well as other Neotropical fishes such as P. brachypomus (Jorge et al., 2018) and M. obtusidens (Villanova et al., 2015).

All the microsatellite loci developed in this work (except Scus11) have PIC values that allow them to be considered highly informative according to the classification proposed by Botstein et al. (1980), and also are higher than those described for Brachyplatystoma rousseauxii (PIC: 0.207-0.910; Batista et al., 2010), Microglanis cottoides (PIC: 0.1150.927, Souza-Shibatta et al., 2013), Steindachneridion parahybae (PIC: 0.429; Fonseca et al., 2016), Hypostomus ancistroides (PIC: 0.089-0.880; Galindo et al., 2015), and Pterygoplichthys pardalis (PIC: 0.294-0.880; Pereira et al., 2012). Moreover, the loci designed for $A$. pardalis and $S$. cuspicaudus showed evidence of linkage equilibrium and most of their allelic frequencies are in Hardy-Weinberg equilibrium, which make them highly informative for determining the diversity and structure of populations of these species. In contrast, most of the loci of P. grosskopfii showed allelic frequencies deviated from the Hardy-Weinberg equilibrium and two pairs of loci showed signals of linkage disequilibrium. It remains to be explored if these characteristics are technical 
problems or a consequence of the high levels of exploitation of $P$. grosskopfii. The linkage disequilibrium has been described in some pairs of loci for the exploited species B. rousseauxii (Batista et al., 2010), S. parahybae (Fonseca et al., 2016), and Pimelodus maculatus (Paiva, Kalapothakis, 2008).

Despite the high frequency of the 2-mer motifs, the microsatellite loci selected in this work were preferentially perfect repeats of 3-mer, 4-mer, and 5-mer motifs; because these type of motifs (uninterrupted and longer monomer) are most recommended in practice, due to their ease in genotyping and classifying the alleles (Gusmão et al., 2006; Castoe et al., 2010, 2012; Guichoux et al., 2011). Contrary to the expectation for longer repeat motifs, the average number of alleles/locus found for $A$. pardalis, P. grosskopfii, and $S$. cuspicaudus is higher than those 2-mer microsatellite loci found in other Neotropical catfishes (Revaldaves et al., 2005; Moeser, Bermingham, 2005; Paiva, Kalapothakis, 2008; Rodrigues et al., 2009, 2015; Carrillo-Ávila et al., 2009; Zamudio et al., 2009; Batista et al., 2010; Telles et al., 2010; Agostini et al., 2011; Carvalho, Beheregaray, 2011; SauloMachado et al., 2011; Muñoz-Rojas et al., 2012; Pereira et al., 2012; Souza et al., 2012; Souza-Shibatta et al., 2013; Prado et al., 2014; Galindo et al., 2015; Ojeda et al., 2016).

Levels of observed and expected heterozygosity for $A$. pardalis and S. cuspicaudus are higher than those reported in microsatellite loci developed for other Neotropical catfishes (Moeser, Bermingham, 2005; Revaldaves et al., 2005; Paiva and Kalapothakis, 2008; Rodrigues et al., 2009, 2015; Carrillo-Ávila et al., 2009; Zamudio et al., 2009; Batista et al., 2010; Telles et al., 2010; Agostini et al., 2011; Carvalho, Beheregaray, 2011; Saulo-Machado et al., 2011; Souza et al., 2012; Muñoz-Rojas et al., 2012; Pereira et al., 2012; Souza-Shibatta et al., 2013; Prado et al., 2014; Ojeda et al., 2016). Additionally, the microsatellite loci identified in this work for P. grosskopfii showed average values of alleles/locus and expected heterozygosity greater than those previously designed for this species by Hernandez-Escobar et al. (2011). Thus, we recommend the loci developed in this work for future population genetic studies and monitoring of populations and stocks of $A$. pardalis, P. grosskopfii, and S. cuspicaudus required in different conservation measures for these species.

\section{ACKNOWLEDGMENTS}

This work was funded by the Universidad Nacional de Colombia, Sede Medellín and Empresas Públicas de Medellín, Grant CT-2013-002443 "Variación genotípica y fenotípica de poblaciones de especies reófilas presentes en el área de influencia del proyecto hidroeléctrico Ituango". We thank the engineering consulting company Integral S.A., for providing the field samples used in this work.

\footnotetext{
- Agostini C, Agudelo PA, Bâ K, Barber PA, Bisol PM, Brouat C et al. Permanent Genetic Resources added to Molecular Ecology Resources Database 1 October 2010-30 November 2010. Mol Ecol Resour. 2011; 11(2):418-21. http://dx.doi. org/10.1111/j.1755-0998.2010.02970.x
}

\author{
- Allendorf FW, Hohenlohe PA, \\ Luikart G. Genomics and the future of \\ conservation genetics. Nat Rev Genet. \\ 2010; 11(10):697-709. http://dx.doi. \\ org/10.1038/nrg2844
}


- Barbará T, Palma-Silva C, Paggi GM, Bered F, Fay MF, Lexer C. Cross-species transfer of nuclear microsatellite markers: Potential and limitations. Mol Ecol. 2007; 16(18):3759-67. http://dx.doi.org/10.1111/ j.1365-294X.2007.03439.x

- Batista JDS, Farias IP, Formiga-Aquino $\mathrm{K}$, Sousa ACB, Alves-Gomes JA. DNA microsatellite markers for "dourada" (Brachyplatystoma rousseauxii, Siluriformes: Pimelodidae), a migratory catfish of utmost importance for fisheries in the Amazon: Development, characterization and inter-specific amplification. Conserv Genet Resour. 2010; 2(1):5-10. http://dx.doi. org/10.1007/s12686-009-9117-5

- Blacket MJ, Robin C, Good RT, Lee SF, Miller AD. Universal primers for fluorescent labelling of PCR fragmentsan efficient and cost-effective approach to genotyping by fluorescence. Mol Ecol Resour. 2012; 12(3):456-63. http://dx.doi. org/10.1111/j.1755-0998.2011.03104.x

- Botstein D, White RL, Skolnick M, Davis RW. Construction of a genetic linkage map in man using restriction fragment length polymorphisms. Am J Hum Genet. 1980; 32(3):314-31.

- Carrillo-Ávila M, Resende EK, Marques DKS, Galetti Jr. PM. Isolation and characterization of polymorphic microsatellites in the threatened catfish Jaú, Zungaro jahu (Siluriformes, Pimelodidae). Conserv Genet. 2009; 10(5):1597-99. http:// dx.doi.org/10.1007/s10592-008-9802-z

- Carvalho DC, Beheregaray LB. Rapid development of microsatellites for the endangered Neotropical catfish Conorhynchus conirostris using a modest amount of 454 shot-gun pyrosequencing. Conserv Genet Resour. 2011; 3(2):373-75. http://dx.doi.org/10.1007/s12686-010-9365-4

- Castoe TA, Poole AW, Gu W, Jason de Koning AP, Daza JM, Smith EN et al. Rapid identification of thousands of copperhead snake (Agkistrodon contortrix) microsatellite loci from modest amounts of 454 shotgun genome sequence. Mol Ecol Resour. 2010; 10(2):341-47. http://dx.doi. org/10.1111/j.1755-0998.2009.02750.x

- Castoe TA, Poole AW, Jason de Koning AP, Jones KL, Tomback DF, Oyler-McCance

SJ et al. Rapid microsatellite identification from illumina paired-end genomic sequencing in two birds and a snake. PLoS One. 2012; 7(2):e30953. http://dx.doi. org/10.1371/journal.pone.0030953
- Edwards YJK, Elgar G, Clark MS, Bishop MJ. The identification and characterization of microsatellites in the compact genome of the Japanese Pufferfish, Fugu rubripes: Perspectives in functional and comparative genomic analyses. J Mol Biol. 1998; 278(4):843-54. http://dx.doi.org/10.1006/ jmbi.1998.1752

- Ekblom R, Galindo J. Applications of next generation sequencing in molecular ecology of non-model organisms. Heredity (Edinb). 2011; 107(1):1-15. http://dx.doi. org/10.1038/hdy.2010.152

- Excoffier L, Lischer HEL. Arlequin suite ver 3.5: A new series of programs to perform population genetics analyses under Linux and Windows. Mol Ecol Resour. 2010; 10(3):564-67. http://dx.doi. org/10.1111/j.1755-0998.2010.02847.x

- Fernandez-Silva I, Whitney J, Wainwright B, Andrews KR, YlitaloWard H, Bowen BW et al. Microsatellites for Next-Generation Ecologists: A PostSequencing Bioinformatics Pipeline. PLoS One. 2013; 8(2):e55990. http://dx.doi. org/10.1371/journal.pone.0055990

- Fonseca FS, Alves Vilar J, Leite AC, Ojeda AP, Caneppele D, Hilsdorf AWS. Development and characterization of microsatellite markers for the critically endangered Neotropical catfish Steindachneridion parahybae. [Electronic supplememtary material 12686_2016_635_ MOESM3_ESM.Zip]. In: Ojeda AP, Hilsdorf AWS, Leite AC, Yang A, Izuno A, He C, et al. Microsatellite records for Volume 8, Issue 4. Conserv Genet Resour. 2016; 8(4):587-94.

- Frankham R. Where are we in conservation genetics and where do we need to go? Conserv Genet. 2010; 11(2):661-63. http://dx.doi.org/10.1007/ s10592-009-0010-2

- Galindo BA, Ferreira DG, Almeida FS, Carlsson J, Sofia SH. Isolation and characterization of 13 polymorphic microsatellite loci in Hypostomus ancistroides (Teleostei, Loricariidae) and cross-amplification in related species. J Fish Biol. 2015; 86(6):1860-66. http://dx.doi. org/10.1111/jfb.12675

- Galvis G, Mojica JI. The Magdalena River fresh water fishes and fisheries. Aquat Ecosyst Health Manag. 2007; 10(2):127-39. http://dx.doi. org/10.1080/14634980701357640 
- Guichoux E, Lagache L, Wagner S, Chaumeil P, Léger P, Lepais 0 et al. Current trends in microsatellite genotyping. Mol Ecol Resour. 2011; 11(4):591-611. http://dx.doi.org/10.1111/ j.1755-0998.2011.03014.x

- Gusmão L, Butler JM, Carracedo A, Gill P, Kayser M, Mayr WR et al. DNA Commission of the International Society of Forensic Genetics (ISFG): An update of the recommendations on the use of Y-STRs in forensic analysis. Forensic Sci Int. 2006; 157(2-3):187-97. http://dx.doi.org/10.1016/j. forsciint.2005.04.002

- Hamilton MB, Pincus EL, Di Fiore A, Fleischer RC. Universal linker and ligation procedures for construction of genomic DNA libraries enriched for microsatellites. Biotechniques. 1999; 27(3):500-07. http:// dx.doi.org/10.2144/99273st03

- Hernandez-Escobar C, Carrillo-Avila M, Ostos-Alfonso H, Valbuena R, OliveraÁngel M, Galetti Jr. PM. Isolation and characterization of microsatellite loci of the neotropical migratory catfish Pimelodus grosskopfii (Siluriformes : Pimelodidae). In: Agostini C, Agudelo PA, Bâ K, Barber PA, Bisol PM, Brouat C, et al. Permanent Genetic Resources added to Molecular Ecology Resources Database 1 October 2010-30 November 2010. Mol Ecol Resour. 2011; 11(2):418-21.

- Jorge PH, Mastrochirico-Filho VA, Hata ME, Mendes NJ, Ariede RB, de Freitas MV et al. Genetic characterization of the fish Piaractus brachypomus by microsatellites derived from transcriptome sequencing. Front Genet. 2018; 9(46):1-12. http://dx.doi. org/10.3389/fgene.2018.00046

- Kumar G, Kocour M. Applications of next-generation sequencing in fisheries research: A review. Fish Res. 2017; 186:11-22. http://dx.doi.org/10.1016/j. fishres.2016.07.021

- Landínez-García RM, Marquez EJ. Microsatellite loci development and population genetics in Neotropical fish Curimata mivartii (Characiformes: Curimatidae). PeerJ. 2018; 6:e5959. http:// dx.doi.org/10.7717/peerj.5959

- Landínez-García RM, Márquez EJ. Development and characterization of 24 polymorphic microsatellite loci for the freshwater fish Ichthyoelephas longirostris (Characiformes: Prochilodontidae). PeerJ. 2016; 4:e2419. http://dx.doi.org/10.7717/ peerj. 2419
- Luo W, Nie Z, Zhan F, Wei J, Wang W, Gao Z. Rapid Development of Microsatellite Markers for the Endangered Fish Schizothorax biddulphi (Günther) Using Next Generation Sequencing and CrossSpecies Amplification. Int J Mol Sci. 2012; 13(12):14946-55. http://dx.doi.org/10.3390/ ijms131114946

- Marshall TC, Slate J, Kruuk LEB, Pemberton JM. Statistical confidence for likelihood-based paternity inference in natural populations. Mol Ecol. 1998; 7(5):639-55. http://dx.doi.org/10.1046/ j.1365-294x.1998.00374.x

- Meglécz E, Nève G, Biffin E, Gardner MG. Breakdown of phylogenetic signal: A survey of microsatellite densities in 454 shotgun sequences from 154 non model Eukaryote species. PLoS One. 2012; 7(7):e40861. http://dx.doi.org/10.1371/ journal.pone.0040861

- Miller AD, Good RT, Coleman RA, Lancaster ML, Weeks AR. Microsatellite loci and the complete mitochondrial DNA sequence characterized through next generation sequencing and de novo genome assembly for the critically endangered orange-bellied parrot, Neophema chrysogaster. Mol Biol Rep. 2013; 40(1):35-42. http://dx.doi.org/10.1007/ s11033-012-1950-z

- Moeser AA, Bermingham E. Isolation and characterization of eight microsatellite loci for the Neotropical freshwater catfish Pimelodella chagresi (Teleostei: Pimelodidae). Mol Ecol Notes. 2005; 5(2):363-65. http://dx.doi.org/10.1111/ j.1471-8286.2005.00928.x

- Mohindra V, Singh A, Barman AS, Tripathi R, Sood N, Lal KK. Development of EST derived SSRs and SNPs as a genomic resource in Indian catfish, Clarias batrachus. Mol Biol Rep. 2012; 39(5):592131. http://dx.doi.org/10.1007/s11033-0111404-z

- Mojica JI, Usma-Oviedo JS, ÁlvarezLeón R, Lasso CA. Libro Rojo de peces dulceacuicolas de Colombia 2012. Bogotá, D.C. Colombia: Instituto de Investigación de los Recursos Biológicos Alexander von Humboldt, Instituto de Ciencias Naturales de la Universidad Nacional de Colombia, WWF Colombia, Universidad de Manizales; 2012. 
- Muñoz-Rojas P, Quezada-Romegialli C, Véliz D. Isolation and characterization of ten microsatellite loci in the catfish Trichomycterus areolatus (Siluriformes: Trichomycteridae), with cross-amplification in seven Trichomycterinae species. Conserv Genet Resour. 2012; 4(2):443-45. http:// dx.doi.org/10.1007/s12686-011-9569-2

- Ojeda AP, Hilsdorf AWS, Leite AC, Yang A, Izuno A, He C et al. Microsatellite records for Volume 8, Issue 4. Conserv Genet Resour. 2016; 8(4):587-94. http:// dx.doi.org/10.1007/s12686-016-0635-7

- Van Oosterhout C, Hutchinson WF, Wills DPM, Shipley P. MICRO-CHECKER: Software for identifying and correcting genotyping errors in microsatellite data. Mol Ecol Notes. 2004; 4(3):535-38. http://dx.doi.org/10.1111/j.14718286.2004.00684.x

- Paiva ALB, Kalapothakis E. Isolation and characterization of microsatellite loci in Pimelodus maculatus (Siluriformes: Pimelodidae). Mol Ecol Resour. 2008; 8(5):1078-80. http://dx.doi.org/10.1111/ j.1755-0998.2008.02160.x

- Peakall R, Smouse PE. GenALEx 6.5: Genetic analysis in Excel. Population genetic software for teaching and research-an update. Bioinformatics. 2012; 28(19):2537-39. http://dx.doi.org/10.1093/ bioinformatics/bts460

- Pereira LH, Hanner R, Foresti F, Oliveira C. Can DNA barcoding accurately discriminate megadiverse Neotropical freshwater fish fauna? BMC Genet. 2013; 14:20. http://dx.doi.org/10.1186/1471-215614-20

- Pereira RP, dos Santos CH dos A, Nascimento PRM, Clímaco GT, Sousa ACB, Campos T et al. Isolation of microsatellite loci in the Amazon sailfin catfish Pterygoplichlhys pardalis (Castelneau, 1855) (Teleostei: Loricariidae). Conserv Genet Resour. 2012; 4(4):889-91. http://dx.doi.org/10.1007/s12686-012-9666-x

- Prado FD, Pardo BG, Guerra-Varela J, Senhorini JA, Martínez P, Foresti F et al. Development and characterization of 16 microsatellites for the Neotropical catfish Pseudoplatystoma reticulatum and cross species analysis. Conserv Genet Resour. 2014; 6(3):679-81. http://dx.doi.org/10.1007/ s12686-014-0180-1
- Primmer CR, Painter JN, Koskinen MT, Palo JU, Merilä J. Factors affecting avian cross-species microsatellite amplification. J Avian Biol. 2005; 36(4):34860. http://dx.doi.org/10.1111/j.09088857.2005.03465.x

- Reis RE, Albert JS, Di Dario F, Mincarone MM, Petry P, Rocha LA. Fish biodiversity and conservation in South America. J Fish Biol. 2016, 89:12-47. http://dx.doi. org/10.1111/jfb.13016

- Restrepo-Escobar N, Alzate JF, Márquez EJ. Mitochondrial genome of the Neotropical catfish Ageneiosus pardalis, Lütken 1874 (Siluriformes, Auchenipteridae). Mitochondrial DNA. 2016a; 27(3):2176-77.

- Restrepo-Escobar N, Alzate JF, Márquez EJ. Mitochondrial genome of the TransAndean shovelnose catfish Sorubim cuspicaudus (Siluriformes,Pimelodidae). Mitochondrial DNA. 2016b; 27(6):3964-65. http://dx.doi.org/10.3109/19401736.2014.9 89506

- Revaldaves E, Pereira LHG, Foresti F, Oliveira C. Isolation and characterization of microsatellite loci in Pseudoplatystoma corruscans (Siluriformes: Pimelodidae) and cross-species amplification. Mol Ecol Notes. 2005; 5(3):463-65. http://dx.doi.org/10.3109/ 19401736.2014.982613

- Rice WR. Analyzing Tables of Statistical Tests. Evolution (NY). 1989; 43:223-25. http://dx.doi.org/10.1111/j.1558-5646.1989. tb04220.x

- Rodrigues FC, Farias IP, Batista JS, AlvesGomes JA. Isolation and characterization of microsatellites loci for "piramutaba" (Brachyplatystoma vaillantii, Siluriformes: Pimelodidae), one of the commercially most important migratory catfishes in the Amazon Basin. Conserv Genet Resour. 2009; 1(1):365-68. http://dx.doi.org/10.1007/ s12686-009-9084-x

- Rodrigues MDN, Moreira CGA, Gutierrez HJP, Almeida DB, Streit Jr. D, Moreira LM. Development of microsatellite markers for use in breeding catfish, Rhamdia sp. African J Biotechnol. 2015; 14(5):400-11. http://dx.doi.org/10.5897/ AJB2014.14116 
- Saarinen EV, Austin JD. When technology meets conservation: Increased microsatellite marker production using 454 genome sequencing on the endangered okaloosa darter (Etheostoma okaloosae). J Hered. 2010; 101(6):784-88. http://dx.doi. org/10.1093/jhered/esq080

- Saulo-Machado AC, Formiga KM, Ortiz MF, Sousa ACB, Alves-Gomes JA, Batista JDS. Polymorphic microsatellite DNA markers for the Amazonian catfish Pseudoplatystoma punctifer (Siluriformes: Pimelodidae). Conserv Genet Resour. 2011; 3(2):307-10. http://dx.doi.org/10.1007/ s12686-010-9349-4

- Schwartz MK, Luikart G, Waples RS. Genetic monitoring as a promising tool for conservation and management. Trends Ecol Evol. 2007; 22(1):25-33. http://dx.doi. org/10.1016/j.tree.2006.08.009

- Somridhivej B, Wang S, Sha Z, Liu H, Quilang J, Xu P et al. Characterization, polymorphism assessment, and database construction for microsatellites from BAC end sequences of channel catfish (Ictalurus punctatus): A resource for integration of linkage and physical maps. Aquaculture. 2008; 275:76-80. http://dx.doi.org/10.1016/j. aquaculture.2008.01.013

- Souza-Shibatta L, Ferreira DG, Oliveira C, De Almeida FS, Shibatta OA, Sofia SH. Development and characterization of microsatellite loci of Microglanis cottoides (Siluriformes: Pseudopimelodidae) and cross-species amplification. Neotrop Ichthyol. 2013; 11(3):581-85. http://dx.doi. org/10.1590/S1679-62252013000300011

- Souza CA, Hashimoto DT, Pereira LHG, Oliveira C, Foresti F, Porto-Foresti F. Development and characterization of microsatellite loci in Phractocephalus hemioliopterus (Siluriformes: Pimelodidae) and their cross-species amplification in six related species. Conserv Genet Resour. 2012; 4(2):499-501. http://dx.doi. org/10.1007/s12686-011-9584-3

- Telles MPC, Resende LV, Brondani RP, Collevatti RG, Costa MC, Silva Júnior NJ. Isolation and characterization of microsatellite markers in the armored catfish Hypostomus gymnorhynchus (Loricariidae). Genet Mol Res. 2010; 9(3):1770-74. http://dx.doi.org/10.4238/ vol9-3gmr868
- Usma-Oviedo JS, Valderrama M, Escobar MD, Ajiaco-Martínez RE, Villa-Navarro FA, Castro F et al. Peces dulceacuícolas migratorios en Colombia. In: Higgins M Lou, Amaya Espinel JD, editors. Plan nacional de las especies migratorias diagnostico e identificacion de acciones para la conservación y el manejo sostenible de las especies migratorias de la biodiversidad Colombiana. Ministerio de ambiente, vivienda y desarrollo territorial, WWF Colombia; 2009. p.103-32.

- Villa-Navarro F, Usma S, Mesa-Salazar L, Sanchez-Duarte P. Pimelodus grosskopfii. The IUCN red list of threatened species 2016: eT49829828A61473588 [Internet]. Available from: http://dx.doi.org/10.2305/IUCN. UK.2016-1.RLTS.T49829828A61473588.en

- Villanova GV., Vera M, Díaz J, Martinez P, Calcaterra NB, Arranz SE. Isolation and characterization of 20 polymorphic microsatellite loci in the migratory freshwater fish Leporinus obtusidens (Characiformes: Anostomidae) using 454 shotgun pyrosequencing. J Fish Biol. 2015; 86(3):1209-17. http://dx.doi.org/10.1111/ jfb.12632

- Yazbeck GM, Oliveira RS, Ribeiro JM, Graciano RD, Santos RP, Carmo FMS et al. A broad genomic panel of microsatellite loci from Brycon orbignyanus (Characiformes: Bryconidae) an endangered migratory Neotropical fish. Sci Rep. 2018; 8(1):8511. http://dx.doi. org/10.1038/s41598-018-26623-x

- Yue G-H, Kovacs B, Orban L. A new problem with cross-species amplification of microsatellites: generation of nonhomologous products. Zool Res. 2010; 31(2):131-40. http://dx.doi.org/10.3724/ SP.J.1141.2010.02131

- Zalapa JE, Cuevas H, Zhu H, Steffan S, Senalik D, Zeldin E et al. Using nextgeneration sequencing approaches to isolate simple sequence repeat (SSR) loci in the plant sciences. Am J Bot. 2012; 99(2):193-208. http://dx.doi.org/10.3732/ ajb.1100394

- Zamudio KR, Robertson JM, Chan LM, Sazima I. Population structure in the catfish Trichogenes longipinnis: Drift offset by asymmetrical migration in a tiny geographic range. Biol J Linn Soc. 2009; 97(2):259-74. http://dx.doi.org/10.1111/ j.1095-8312.2009.01209.x 


\section{Neotropical |chthyology}

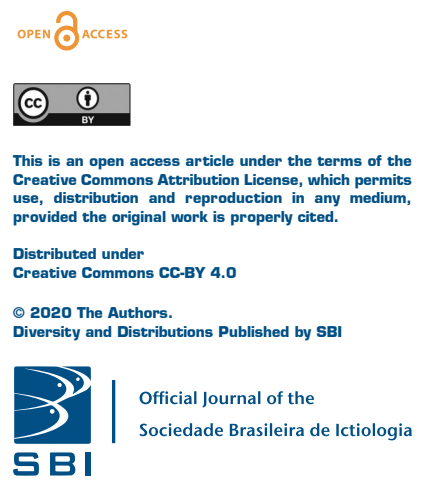

\section{- Zhang J, Ma W, Song X, Lin Q, Gui JF,}

Mei J. Characterization and development of EST-SSR markers derived from transcriptome of yellow catfish. Molecules. 2014; 19(10):16402-15. http://dx.doi.

org/10.3390/molecules191016402

\section{REFERENCES}

\section{AUTHOR CONTRIBUTIONS}

Natalia Restrepo-Escobar: Conceptualization, Data curation, Formal Analysis, Funding acquisition, Investigation, Methodology, Validation, Visualization, Writing (original draft), Writing (review \& editing). Edna J. Márquez: Conceptualization, Data curation, Formal Analysis, Funding acquisition, Investigation, Methodology, Project administration, Resources, Supervision, Validation, Visualization, Writing (original draft), Writing (review \& editing).

\section{ETHICAL STATEMENTS}

Samples were collected under the environmental license \# 0155 of January 30, 2009 from Ministerio de Ambiente, Vivienda y Desarrollo Territorial.

\section{COMPETING INTERESTS}

Not applicable.

\section{HOW TO CITE THIS ARTICLE}

- Restrepo-Escobar N, Márquez EJ. Microsatellite loci development for three catfish species from northwestern South America. Neotrop Ichthyol. 2020; 18(1):e190079. https://doi. org/10.1590/1982-0224-2019-0079 
